\title{
Fault Detection for Wheeled Mobile Robots with Parametric Uncertainty*
}

\author{
${ }^{\dagger}$ W. E. Dixon, I. D. Walker, and D. M. Dawson \\ ${ }^{\dagger}$ Oak Ridge National Laboratory \\ Department of Electrical \& Computer Engineering \\ Bldg. 7601, Mailstop 6305 \\ P.O. Box 2008 \\ Clemson University \\ Clemson, SC 29634-0915 \\ Oak Ridge, TN 37831 \\ dixonwe@ornl.gov \\ email: ddawson, ianw@ces.clemson.edu
}

\begin{abstract}
In this paper, we develop a new method for Wheeled Mobile Robot (WMR) fault detection. Specifically, we develop kinematic and dynamic models of the WMR in the presence of faults such as a change in the wheel radius (e.g., deformation, broken spoke, flat tire) or general kinematic disturbances that model slipping or skidding faults. Utilizing the WMR models, we employ a torque filtering technique to develop a prediction error based fault detection residual. The structure of the prediction error allows for fault detection despite parametric uncertainty in the WMR model.
\end{abstract}

\section{Introduction}

Wheeled mobile robots (WMRs) have been employed for applications including: military operations, surveillance, security, mining operations, planetary exploration, entertainment, aids for mobility impaired humans, and materials handling, transport, and inspection. As described above, many WMR applications require interaction with humans, handling of volatile materials, and/or operation in remote and hazardous environments, such as found in space and radioactive applications; hence, reliability and safety are of paramount concern. Based on the importance of reliability and safe operation of WMRs, several researchers have recently investigated WMR reliability and fault tolerance.

To develop mobile robotic systems that are tolerant to faults some researchers have proposed utilizing a multiple mobile robot scheme (see $[7,8]$ and the references within). Given the detection of a fault, the system degrades gracefully by reconfiguring the formation to compensate for the failed WMR. In addition to mobile robot redundancy, researchers have also investigated redundant sensing tech-

${ }^{*}$ This research is supported by the Eugene P. Wigner Fellowship for a Fellow and staff member at the Oak Ridge National Laboratory, managed by UT-Battelle, LLC, for the U.S. Department of Energy under contract DE-AC05-00OR22725. niques which allow a system to switch to "healthy" sensors following a sensor failure (see $[11,12]$ and the references within). Each of the above redundancy approaches can only be exploited if the fault detection is effective: hence, mobile robot fault detection has become an issue of significant interest.

In [8], Parker utilizes the concept of multiple mobile robots to develop a fault tolerant system. Specifically, each of the robots exploits the concept of motivational behaviors such as impatience and acquience to determine if a fault has occurred in a cooperative partner. Based on the detection of the fault, the remaining functional cooperative partners can work to accommodate the robot failure. Although the concept of motivational behavior as a fault detection tool is beneficial from the viewpoint that a fault can be detected by another robot, it lacks sensitivity, resulting in a relatively slow response to the failure. That is, the time to detect the fault is a function of the task the robot is performing with respect to other robots in the system.

Another approach that has been utilized to target fault detection in mobile robots is the use of Kalman filters (see $[5,9,10,15])$. As described in [9], the overall philosophy of the Kalman filtering method is to exploit analytical redundancy by thresholding the residual generated by the difference between the measured values and values predicted by the Kalman filter (based on certain assumptions regarding the system model). In [9], Romoumeliotis et al. utilize a Kalman filtering technique to detect and identify actuator faults such as flat tires and a periodic bump in the wheel. In [10], the work in [9] is extended to detect and identify faults in the left wheel, right wheel, and heading angular velocity measurements; unfortunately, the algorithm utilized in $[9,10]$ to threshold the residuals is not described. More recently, in [5] Goel et al. utilize a similar Kalman filtering technique to detect the sensor and actuator faults investigated in $[9,10]$. Once the fault is detected, Goel et al. utilize a backpropogation Neural Network structure to process the residual set to identify the fault. In [15], Washington also utilizes a method that is based on a combination of continuous and discrete state 
estimation, Kalman filters and a Markov model representation to detect and identify actuator faults occurring in the WMR (e.g., overcurrents in the wheel motors).

Based on the review of mobile robot fault detection literature given above, it is clear that none of the aforementioned research has incorporated the nonlinear dynamic model of the mobile robot in the fault detection algorithm, and hence, the effects of uncertainty in the mechanical parameters that are used to complete the dynamic model (e.g., payload mass, friction, etc.) have not been investigated. From a review of fault detection literature that targets robot manipulators (see $[2,4,13,14,17])$, it is clear that the key difficulty endemic to manipulator fault detection (and hence, similar electromechanical systems such as WMRs) is that the normal (fault-free) dynamics of the robot lead to inevitable deviations from the nominal trajectory in fault-free operation, and the magnitude of these deviations cannot be predicted (and therefore can appear to be a fault unless properly masked by the thresholds), when the dynamics are not explicitly considered in the analysis. Clearly, fault detection will be most effective when good dynamic models for the system are considered in the fault detection tests (residuals) or the threshold selection, or both.

In this paper, we build on the research presented in [2] to develop a new method for WMR fault detection. Specifically, we develop kinematic and dynamic models of the WMR in the presence of actuator faults such as a change in the wheel radius (e.g., deformation, broken spoke, flat tire) or general kinematic disturbances that model slipping or skidding faults. The approach is based on the generation of a residual and exploits the structure of the full nonlinear dynamics of the WMR through a filtered torque estimate that does not rely upon the measurement of acceleration quantities (unlike many of the model-based fault detection algorithms that are utilized to detect faults in robot manipulators). The fault detection residual is based on a prediction error which is the difference between the filtered torque signal and an estimate of the filtered torque. The structure of the prediction error based fault detection algorithm lends itself to take into account the inevitable uncertainty in the robot parameters. A threshold is developed for the prediction error residual.

The paper is organized as follows. In Section 2 and 3 , we develop the kinematic and dynamic models of the WMR, respectively. In Section 4, the torque filtering technique is described. Section 5 describes how the filtered torque signal can be utilized to generate the prediction error based residual, and concluding remarks are presented in Section 6 .

\section{Kinematic Model}

The kinematic model for a two-whee ${ }^{1}$, differential-drive WMR is assumed to have the following form

$\dot{q}=S(q)\left(r+\delta_{1}\left(t-T_{1}\right)\right)\left[\begin{array}{c}\frac{\omega_{R}+\omega_{L}}{2} \\ \frac{\omega_{R}-\omega_{L}}{D}\end{array}\right]+\left[\begin{array}{c}\delta_{2}\left(t-T_{2}\right) \\ \delta_{3}\left(t-T_{2}\right) \\ \delta_{4}\left(t-T_{2}\right)\end{array}\right]$

where $q(t), \dot{q}(t) \in \mathbb{R}^{3}$ are defined as

$$
q=\left[\begin{array}{lll}
x_{c} & y_{c} & \theta
\end{array}\right]^{T} \quad \dot{q}=\left[\begin{array}{lll}
\dot{x}_{c} & \dot{y}_{c} & \dot{\theta}
\end{array}\right]^{T}
$$

$\dot{x}_{c}(t), \dot{y}_{c}(t), \dot{\theta}(t) \in \mathbb{R}^{1}$ denote the time derivatives of $x_{c}(t), y_{c}(t), \theta(t) \in \mathbb{R}^{1}$ which represent the Cartesian position of the center of mass (COM) (which is assumed to lie at the midpoint of the wheel axis for simplicity) of the WMR along the $X$ and $Y$-coordinate axis of the Cartesian plane and the orientation of the WMR (see Figure 1), respectively, the matrix $S(q) \in \mathbb{R}^{3 \times 2}$ is defined as follows

$$
S(q)=\left[\begin{array}{ll}
\cos \theta & 0 \\
\sin \theta & 0 \\
0 & 1
\end{array}\right]
$$

$r \in \mathbb{R}^{1}$ denotes the constant (pre-fault) radius of the wheels, $\omega_{R}(t), \omega_{L}(t) \in \mathbb{R}^{1}$ represent the angular velocities of the right and left wheels, respectively, $D \in \mathbb{R}^{1}$ represents the length of the axis between the wheels, $\delta_{1}\left(t-T_{1}\right) \in \mathbb{R}^{1}$ represents a fault that occurs at time $T_{1}$ that physically represents a fault in the wheel radius (e.g., deformation, flat tire, etc.), and $\delta_{i}\left(t-T_{2}\right) \in \mathbb{R}^{1}$ $\forall i=2,3,4$, represent faults that occur at time $T_{2}$ that physically represent a fault due to slipping or skidding conditions of the WMR. To simplify the subsequent fault detection algorithm development, we rewrite (1) as follows

$$
\dot{q}=S(q) v+\delta(t-T)
$$

where $v(t) \in \mathbb{R}^{2}$ represents the (pre-fault) linear and angular velocity of the WMR, denoted by $v_{1}(t), v_{2}(t) \in \mathbb{R}^{1}$, respectively, and is defined as follows

$$
v=\left[\begin{array}{l}
v_{1} \\
v_{2}
\end{array}\right]=r\left[\begin{array}{l}
\frac{\omega_{R}+\omega_{L}}{2} \\
\frac{\omega_{R}-\omega_{L}}{D}
\end{array}\right]
$$

and $\delta(t-T) \in \mathbb{R}^{3}$ is defined as

$$
\begin{aligned}
\delta(t-T) \triangleq\left[\begin{array}{c}
\bar{\delta}_{1}(t-T) \\
\bar{\delta}_{2}(t-T) \\
\bar{\delta}_{3}(t-T)
\end{array}\right]=\left[\begin{array}{c}
\delta_{2}\left(t-T_{2}\right) \\
\delta_{3}\left(t-T_{2}\right) \\
\delta_{4}\left(t-T_{2}\right)
\end{array}\right] \\
+\frac{\delta_{1}\left(t-T_{1}\right)}{2}\left[\begin{array}{c}
\left(\omega_{R}+\omega_{L}\right) \cos \theta \\
\left(\omega_{R}+\omega_{L}\right) \sin \theta \\
\frac{2\left(\omega_{R}-\omega_{L}\right)}{D}
\end{array}\right]
\end{aligned}
$$

\footnotetext{
${ }^{1}$ Note that the expression "two-wheel mobile robot" refers to a mobile robot with two active wheels and $n$-castor-like wheels (passive).
} 
where $T$ is a time instant defined as

$$
T=\min \left(T_{1}, T_{2}\right)
$$

The faults given in (6) are assumed to be first-order differentiable and upper bounded as shown below

$$
\left|\bar{\delta}_{i}(t-T)\right| \leq \Delta_{i}, \quad \forall i=1,2,3
$$

where $\Delta_{i} \in \mathbb{R}^{1}$ are positive bounding constants. Furthermore, we note that

$$
\delta(t-T)=0, \quad t<T
$$

and the standard kinematic model for the pure rolling and nonslipping kinematic wheel is recovered.

Remark 1 The model given in (1) does not make a distinction between the left wheel radius and the right wheel radius. Making a distinction between the left wheel and the right wheel radius may be easily incorporated into the model for improved fault identification capabilities; however, since the focus of this paper is the detection of a fault and since the overall structure of the fault detection algorithm will not be altered, we do not make the distinction for the sake of simplicity.

Remark 2 Note that the kinematic model for a WMR subject to the so-called matched disturbance is defined as follows [1]

$$
\dot{q}=S(q) v+\rho_{M}(t)\left[\begin{array}{lll}
\cos \theta & \sin \theta & 0
\end{array}\right]^{T}
$$

where $\rho_{M}(t) \in \mathbb{R}^{1}$ denotes a bounded disturbance. In addition, the kinematic model for a WMR subject to the so-called unmatched disturbance is defined as follows [1]

$$
\dot{q}=S(q) v+\rho_{U}(t)\left[\begin{array}{lll}
\sin \theta & -\cos \theta & 0
\end{array}\right]^{T}
$$

where $\rho_{U}(t) \in \mathbb{R}^{1}$ denotes a bounded disturbance. Note that it is clear from (4), (10), and (11) that the matched disturbance and unmatched disturbance problems are both special cases of the model used in (4).

Remark 3 With regard to the wheel radius fault denoted by $\delta_{1}\left(t-T_{1}\right)$, we assume the following inequality is satisfied

$$
r+\delta_{1}\left(t-T_{1}\right)>0 .
$$

This is, catastrophic faults that result in a zero wheel radius (e.g., the loss of the entire wheel assembly) are not considered.

\section{Dynamic Model}

The dynamic model for a two-wheel, differentially driven WMR can be written in the following form

$$
S^{T} M S \dot{v}+S^{T} M \dot{\delta}+S^{T} F_{d}(S v+\delta)=S^{T} B \tau
$$

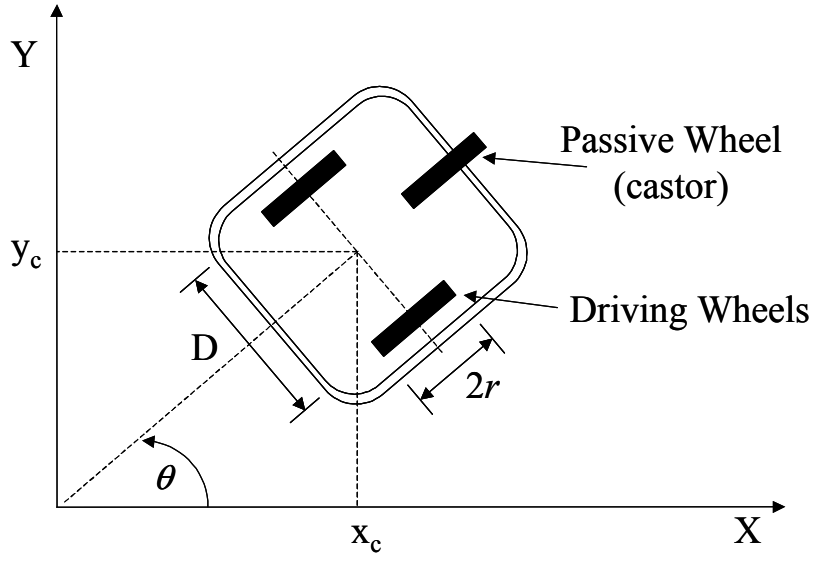

Figure 1: Wheeled Mobile Robot

where $\dot{v}(t) \in \mathbb{R}^{2}$ denotes the time derivative of $v(t)$ defined in (5), $S(q)$ was defined in (3), $M \in \mathbb{R}^{3 \times 3}$ represents the inertia matrix, $F_{d} \in \mathbb{R}^{3 \times 3}$ represents a diagonal matrix of friction coefficients, $\tau(t) \in \mathbb{R}^{2}$ represents the torque input vector, and $B(q) \in \mathbb{R}^{3 \times 2}$ represents an input matrix that governs torque transmission and is defined as follows

$$
B=\frac{1}{\left(r+\delta_{1}\left(t-T_{1}\right)\right)}\left[\begin{array}{cc}
\cos \theta & \cos \theta \\
\sin \theta & \sin \theta \\
D & -D
\end{array}\right] .
$$

To facilitate the development of the subsequent fault detection algorithms, we multiply both sides of (13) by $r+\delta_{1}\left(t-T_{1}\right)$, substitute (4) into (13) for $v(t)$, and then simplify the resulting expression as follows

$$
\left[\begin{array}{cc}
1 & 1 \\
D & -D
\end{array}\right] \tau=r\left(\bar{M} \dot{v}+\bar{F}_{d} v\right)+\zeta(t-T)
$$

where $\zeta(t-T) \in \mathbb{R}^{2 \times 1}$ is defined as follows

$$
\begin{aligned}
\zeta(t-T)= & \delta_{1}\left(t-T_{1}\right)\left(\bar{M} \dot{v}+\bar{F}_{d} v\right) \\
& +\left(r+\delta_{1}\left(t-T_{1}\right)\right) \\
& \left(S^{T} M \dot{\delta}(t-T)+S^{T} F_{d} \delta(t-T)\right)
\end{aligned}
$$

and

$$
\bar{M}=S^{T} M S, \quad \bar{F}_{d}=S^{T} F_{d} S .
$$

Based on the following assumption

$$
\delta_{1}\left(t-T_{1}\right), \delta(t-T), \text { and } \dot{\delta}(t-T)=0 \quad \forall t<T
$$

it is clear that

$$
\zeta(t-T)=0 \quad \forall t<T .
$$

That is, in the absence of a fault the typical dynamic model for the pure rolling and nonslipping two-wheel, differentially driven WMR is recovered [3].

The dynamic equation given in (15), exhibits the following property [6] which is utilized in conjunction with the following assumptions in the subsequent fault detection algorithm development. 
Property 1: The dynamic model given in (15) can be linearly parameterized as follows

$$
Y(q, v, \dot{v}) \theta_{L}=r\left(\bar{M} \dot{v}+\bar{F}_{d} v\right)
$$

in the absence of faults $($ i.e., $t<T$ ) where $Y(\cdot) \in$ $\mathbb{R}^{n \times p}$ denotes a known regression matrix and $\theta_{L} \in$ $\mathbb{R}^{p}$ contains the unknown constant system parameters.

Assumption 1: Each of the constant system parameters defined in (20) can be lower and upper bounded as indicated by the following inequalities

$$
{\underline{\theta_{L}}}_{j}<\theta_{L j}<\bar{\theta}_{L j}
$$

where $\theta_{L j}$ denotes the $j$-th component of the vector $\theta_{L}$, and $\underline{\theta_{L}}, \bar{\theta}_{L} \in \mathbb{R}^{p}$ denote vectors of known, constant bounds for the unknown parameters.

Assumption 2: A control is designed which ensures that in the absence of a fault (i.e., $t<T$ ) $q(t), \dot{q}(t), v(t), \tau(t) \in \mathcal{L}_{\infty}$ and that $\lim _{t \rightarrow \infty} q(t)=q_{d}(t)$ where $q_{d}(t) \in \mathbb{R}^{n}$ represents the desired trajectory. Note that based on the form of the dynamic model given in (15) and the expression given in (14), if $q(t), \dot{q}(t), \tau(t) \in \mathcal{L}_{\infty}$, it is clear that $\dot{v}(t) \in \mathcal{L}_{\infty}$.

Remark 4 One method for detecting faults in the WMR could be to utilize (15) and (20) to isolate the fault terms as shown below

$$
\zeta(t-T)=\left[\begin{array}{cc}
1 & 1 \\
D & -D
\end{array}\right] \tau-Y(q, v, \dot{v}) \theta_{L} .
$$

Unfortunately, due to the fact that (22) would require exact model knowledge of the system and acceleration measurements, it is clear that (22) is impractical for fault detection purposes; hence, we are motivated to craft a fault detection algorithm that is independent of acceleration measurements and exact knowledge of the system parameters.

\section{Torque Filtering}

Motivated by the desire to eliminate acceleration measurements from the subsequent fault detection algorithm, we define a filtered torque signal denoted by $\tau_{f}(t) \in \mathbb{R}^{2}$ as follows $[6]$

$$
\tau_{f}=f *\left[\begin{array}{cc}
1 & 1 \\
D & -D
\end{array}\right] \tau
$$

where $*$ denotes the standard convolution operation, $\tau(t)$ was defined in (13), the filter function, denoted by $f(t) \in$ $\mathbb{R}^{1}$, is given by

$$
f=\alpha \exp (-\beta t)
$$

and $\alpha, \beta \in \mathbb{R}^{1}$ denote positive filter constants. By substituting the left-side of (15) into (23) for $\tau(t)$ and utilizing standard convolution properties (see Appendix A), we can rewrite (23) in terms of the following linear parameterization

$$
\tau_{f}=Y_{f} \theta_{L}+\zeta_{f}
$$

where $\theta_{L}$ denotes the same unknown, constant parameter vector defined in $(20), Y_{f}(q, v) \in \mathbb{R}^{2 \times p}$ denotes the measurable, filtered regression matrix which is independent of acceleration measurements and is explicitly given by

$$
\begin{aligned}
Y_{f} \theta_{L}= & \dot{f}(t) *\{r \bar{M}(q(t)) v(t)\}+r f(0) M(q(t)) v(t) \\
& -r f(t) M(q(0)) v(0) \\
& +f(t) *\left\{-r\left(\overline{\bar{M}}(q(t)) v(t)-\bar{F}_{d}(q(t) v(t))\right\}\right.
\end{aligned}
$$

and $\zeta_{f}(t-T) \in \mathbb{R}^{2}$ denotes a filtered fault signal that is also independent of acceleration measurements and is defined as follows

$$
\zeta_{f}(t)=f(t) * \zeta(t-T) .
$$

The structure of (25) is utilized in the subsequent analysis; however, since $\theta_{L}$ is a vector of uncertain parameters, the form of the filtered torque signal given by $(25)$ is not implementable. An equivalent, implementable (i.e., a measurable, acceleration independent) form of the filtered torque signal can be determined by utilizing (23) and (24) along with standard Laplace Transform properties to generate the following differential equality

$$
\dot{\tau}_{f}=-\beta \tau_{f}+\alpha\left[\begin{array}{cc}
1 & 1 \\
D & -D
\end{array}\right] \tau \quad \tau_{f}(0)=0
$$

where $\alpha, \beta$ were defined in (24).

Remark 5 Due to the structure of the above torque filtering technique, the filtered version of the fault is delayed. To mitigate the delay, $\beta$ is made increasingly large. Based on (25), it also is clear that the fault can be isolated in terms of an expression that is independent of link acceleration measurements. Thus, we are now motivated to design an algorithm based on (25) that can detect faults in a WMR despite the presence of parametric uncertainty.

\section{Prediction Error Based Fault Detection}

The objective of this paper is to design an algorithm that can detect faults in mobile robots despite uncertainty in the mechanical parameters. To this end, we define a measurable prediction error signal, denoted by $\varepsilon(t) \in \mathbb{R}^{2}$, as follows

$$
\varepsilon=\tau_{f}-\hat{\tau}_{f}
$$

where $\tau_{f}$ was defined in (28), and $\hat{\tau}_{f} \in \mathbb{R}^{2}$ is a subsequently designed filtered torque estimate. This method of residual generation is similar to one of the fault detection tests proposed in [14] for robot manipulators; however, in 
[14] acceleration estimates were required for implementation.

Due to the presence of parametric uncertainty in (13), the filtered torque estimate given in (29) is designed as follows

$$
\hat{\tau}_{f}=Y_{f} \hat{\theta}_{L}
$$

where $\hat{\theta}_{L} \in \mathbb{R}^{p}$ is a constant, best-guess parameter estimate $^{2}$ for $\theta_{L}$ defined in $(20)$ and $Y_{f}(q, \dot{q})$ was defined in (25). From the design of $\hat{\tau}_{f}(t)$, we can use (25), (29), and (30) to obtain a new expression for $\varepsilon(t)$ given as follows

$$
\varepsilon=Y_{f} \tilde{\theta}_{L}+\zeta_{f}
$$

where $\tilde{\theta} \in \mathbb{R}^{p}$ quantifies the constant mismatch between the actual uncertain parameters and the constant, best guess parameter estimate as shown below

$$
\tilde{\theta}_{L}=\theta_{L}-\hat{\theta}_{L}
$$

Based on Assumption 1, we can upper bound the prediction error signal given in (31) as follows

$$
\left|\varepsilon_{i}\right| \leq \rho_{i}(t)+\left|\zeta_{f i}\right|
$$

where $\rho(t) \in \mathbb{R}^{2}$ is a positive bounding signal selected to satisfy the following inequality

$$
\left|\left(Y_{f} \tilde{\theta}_{L}\right)_{i}\right| \leq \rho_{i}(t)
$$

and $(\cdot)_{i}$ represents the $i$-th element of a vector. Based on the structure of (33), we define a fault indicating, deadzone residual function, denoted by $D_{1}[\cdot] \in \mathbb{R}^{1}$, as follows

$$
D_{1}\left[\varepsilon_{i}\right]= \begin{cases}\left|\varepsilon_{i}\right| & \text { if }\left|\varepsilon_{i}\right|>\rho_{i}(t) \\ 0 & \text { if }\left|\varepsilon_{i}\right| \leq \rho_{i}(t)\end{cases}
$$

to determine if a fault occurs. That is, if

$$
D_{1}\left[\varepsilon_{i}\right]>0
$$

then a fault is present in the system; however, if the parameter uncertainty in the system is relatively large, then some faults may not be detected due to the inability of the fault detection scheme given in (35) to distinguish the faults from the parameter uncertainty.

Remark 6 The motivation for selecting (35) as shown below

$$
D_{1}\left[\varepsilon_{i}\right]=\left|\varepsilon_{i}\right| \text { if }\left|\varepsilon_{i}\right|>\rho_{i}(t)
$$

versus some positive constant (i.e., $D_{1}\left[\varepsilon_{i}\right]=1$ if $\left|\varepsilon_{i}\right|>$ $\left.\rho_{i}(t)\right)$, arises from the additional flexibility gained with regard to observing the extent that the residual given in (35) was violated. That is, by utilizing (35), possible false alarm conditions that may occur (e.g., due to signal noise, numeric round-off, etc.) may be avoided.

\footnotetext{
${ }^{2}$ The term best-guess-estimate is utilized to signify a constant parameter estimate that is defined by the user as a best-guess of the actual value of the unknown parameter. Specifically, the user may obtain a value for the best-guess estimate utilizing any of the appropriate parameter identification techniques that are found in literature.
}

Remark 7 If exact model knowledge of the system is available, then we can simply redesign $\hat{\tau}_{f}(t)$ as follows

$$
\hat{\tau}_{f}=Y_{f} \theta_{L}
$$

where $\theta_{L}$ defined in (20) is now assumed to be known. After substituting (25) and (37) into (29), we obtain the following expression for $\varepsilon(t)$

$$
\varepsilon=\zeta_{f}
$$

hence, at least, in the theory, $\varepsilon(t)=0$ for $t<T$. It should be noted that in practice small uncertainties and measurement noise will no doubt ensure that $\varepsilon(t) \neq 0$ for $t<T$ (i.e., $\left\|\varepsilon_{i}(t)\right\|$ will equal some unknown time-varying function); hence, we define a fault indicating, dead-zone residual function, denoted by $D_{2}[\cdot] \in \mathbb{R}^{1}$, as follows

$$
D_{2}\left[\varepsilon_{i}\right]= \begin{cases}\left|\varepsilon_{i}\right| & \text { if }\left|\varepsilon_{i}\right|>\left(\mu_{o}\right)_{i} \\ 0 & \text { if }\left|\varepsilon_{i}\right| \leq\left(\mu_{o}\right)_{i}\end{cases}
$$

such that, if a fault is present in the system

$$
D_{2}\left[\varepsilon_{i}\right]>0
$$

where $\mu_{o} \in \mathbb{R}^{2}$ is a vector of positive, scalar design constants that are experimentally determined to account for small uncertainties and measurement noise.

\section{Conclusion}

In conclusion, this paper provides kinematic and dynamic models of a mobile robot system that is subject to faults such as a change in the wheel radius (due to deformations, flat tires, broken spokes, etc.) and general kinematic disturbances that could physically represent slipping and skidding effects. A prediction error based fault detection algorithm is presented that can be utilized to detect the aforementioned faults despite parametric uncertainty in the dynamic model. In subsequent work, we will 1) further characterize the mobile robot faults in an attempt to develop a fault identification scheme, 2) investigate generalizing the fault to incorporate a broader class of mobile vehicles, 3 ) leverage off of our recent results with fault detection in robot manipulators [2] to develop algorithms that provide increased sensitivity (faster fault detection capabilities) by further mitigating the effects of parametric uncertainty in the system model, and 4) develop an experimental testbed to demonstrate the effectiveness of the fault detection algorithm in the presence of parametric uncertainty.

\section{References}

[1] C. Canudas de Wit and H. Khennouf, "QuasiContinuous Stabilizing Controllers for Nonholonomic Systems: Design and Robustness Considerations", Proceedings of the 3rd European Control Conference, pp. 2630-2635, 1995. 
[2] W. E. Dixon, I.D. Walker, D.M. Dawson, and J. P. Hartranft, "Fault Detection for Robot Manipulators with Parametric Uncertainty: A Prediction Error Based Approach", Proc. IEEE International Conference on Robotics and Automation, pp. 3628-3634, 2000.

[3] W. E. Dixon, D. M. Dawson, F. Zhang, and E. Zergeroglu, "Global Exponential Tracking Control of A Mobile Robot System via a PE Condition", IEEE Transactions on Systems, Man, and Cybernetics Part B: Cybernetics, Vol. 30, No. 1, pp. 129-142, 2000.

[4] B. Freyermuth, "An Approach to Model-Based Fault Diagnosis of Industrial Robots", Proc. IEEE International Conference on Robotics and Automation, Sacramento, CA, pp. 1350-1356, 1991.

[5] P. Goel, G. Dedeoglu, S. I. Roumeliotis, and G. S. Sukhatme, "Fault Detection and Identification in a Mobile Robot Using Multiple Model Estimation and Neural Network", Proceedings of the IEEE International Conference on Robotics and Automation, pp. 2302-2309, 2000.

[6] F. Lewis, C. Abdallah, and D. Dawson, Control of Robot Manipulators, New York: MacMillan Publishing Co., 1993.

[7] D. G. Michaelson and J. Jiang, "Modeling of Redundancy in Multiple Mobile Robots", Proc. of the IEEE American Control Conference, pp. 1083-1087, 2000.

[8] L.E. Parker, "ALLIANCE: An architecture for Fault Tolerant Multirobot Cooperation", IEEE Transactions on Robotics and Automation, Vol. 14, No. 2, pp. 220-240, April 1998.

[9] S. I. Roumeliotis, G. S. Sukhatme, and G. A. Bekey, "Fault Detection and Identification in a Mobile Robot Using Multiple-Model Estimation", Proc. IEEE International Conference on Robotics and Automation, pp. 2223-2228, 1998.

[10] S. I. Roumeliotis, G. S. Sukhatme, and G. A. Bekey, "Sensor Fault Detection and Identification in a Mobile Robot", Proc. IEEE International Conference on Intelligent Robots and Systems, pp. 1383-1388, 1998.

[11] S. Scheding, E. Nebot, and H.Durrant-Whyte, "The Detection of Faults in Navigation Systems: A Frequency Domain Approach", Proc. of the IEEE International Conference on Robotics and Automation, pp. 2217-2222, 1998.

[12] M. Soika, "Grid Based Fault Detection and Calibration of Sensors on Mobile Robots", Proc. IEEE International Conference on Robotics and Automation, Albuquerque, NM, pp. 2589-2594, 1997.
[13] A. Vemuri and M. M. Polycarpou, "Neural-NetworkBased Robust Fault Diagnosis in Robotic Systems", IEEE Transactions on Neural Networks, Vol. 8., No. 6, pp. 1410-1419, 1997.

[14] M.L. Visinsky, J.R. Cavallaro, and I.D. Walker, "A Dynamic Fault Tolerance Framework for Remote Robots", IEEE Transactions on Robotics and Automation, Vol. 11, No. 4, pp. 477-490, 1995.

[15] R. Washington, "On-Board Real-Time State and Fault Identification for Rovers", Proceedings of the IEEE International Conference on Robotics and Automation, pp. 1175-1181, 2000.

[16] J Wunnenberg and P.M. Frank, "Dynamic Model Based Incipient Fault Detection Concept for Robots", Proc. IFAC 11th Triennial World Congress, Tallinn, Estonia, USSR, pp. 61-66, 1990.

[17] F. Zanaty, "Consistency Checking Techniques for the Space Shuttle Remote Manipulator System", SPAR Journal of Engineering and Technology, Vol. 2, No. 1, pp. 40-49, 1993.

\section{Appendix A}

In order to rewrite (23) in terms of the linear parameterization given in (25), we first note that (13) can be written in the following form [6]

$$
\left[\begin{array}{cc}
1 & 1 \\
D & -D
\end{array}\right] \tau=\dot{h}+g
$$

where

$$
\dot{h}=\frac{d}{d t}(r \bar{M}(q(t)) v(t))
$$

and

$$
g=-r\left(\dot{\bar{M}}(q(t)) v(t)-\bar{F}_{d}(q(t) v(t))+\zeta(t-T) .\right.
$$

After substituting (41) into (23), we obtain the following expression

$$
\begin{aligned}
\tau_{f}= & \dot{f}(t) *\{r \bar{M}(q(t)) v(t)\} \\
& +r f(0) M(q(t)) v(t)-r f(t) M(q(0)) v(0) \\
& +f(t) *\left\{-r\left(\dot{\bar{M}}(q(t)) v(t)-\bar{F}_{d}(q(t) v(t))\right.\right. \\
& +\zeta(t-T)\}
\end{aligned}
$$

where the facts that

$$
f *\{\dot{h}+g\}=f * \dot{h}+f * g
$$

and

$$
f * \dot{h}=\dot{f} * h+f(0) h-f h(0)
$$

have been utilized. Hence, based on (44), it is straightforward to conclude that (23) can be rewritten in the structure given in (25). 